\title{
Intralesional Methotrexate for the Treatment of Advanced Keratinocytic Tumors: A Multi-Center Retrospective Study
}

\author{
Giulio Gualdi - Simone Caravello (1) - Fabiano Frasci - Federica Giuliani - Ruggero Moro • Maria C. Fargnoli • \\ Valeria Ciciarelli · Giuseppe Argenziano - Caterina M. Giorgio · Celia Requena · Eduardo Nagore · \\ Onofre Sanmartín · Antonio Martorell · Fabrizio Fantini · Piergiacomo Calzavara-Pinton · Paolo Amerio
}

Received: April 10, 2020 / Published online: June 13, 2020

(C) The Author(s) 2020

\section{ABSTRACT}

Introduction: Keratinocyte tumors (KT) are frequently observed. Surgery is the treatment gold standard. In some cases, a surgical approach might not be the best option. Radiotherapy (RT) and systemic treatments can frequently cause side effects or be contraindicated.

Digital Features To view digital features for this article go to https://doi.org/10.6084/m9.figshare.12320330

G. Gualdi · F. Giuliani · P. Amerio

Department of Medicine and Aging Science,

Dermatologic Clinic, University G. D'Annunzio,

Chieti Pescara, Italy

S. Caravello $(\varangle) \cdot$ P. Calzavara-Pinton

Department of Dermatology, Spedali Civili di

Brescia, University of Brescia, Brescia, Italy

e-mail: caravello.simone29@gmail.com;

s.caravello001@unibs.it

F. Frasci

University of Sassari, Sassari, Italy

R. Moro

Escuela de Doctorado, Universidad Católica de

Valencia San Vicente Mártir, València, Spain

R. Moro

Dermatologia, Clinica San Carlo, Paderno Dugnano, Italy

M. C. Fargnoli · V. Ciciarelli

Dermatology, Department of Biotechnological and Applied Clinical Sciences, University of L'Aquila,

L'Aquila, Italy
Intralesional methotrexate (MTX) can be a conservative yet effective alternative. We decided to evaluate the effectiveness and safety of intralesional chemotherapy with MTX for the treatment of squamous cell carcinoma (SCC), keratoacanthoma (KA), and basal cell carcinoma (BCC).

Methods: All patients had a histologically confirmed diagnosis of BCC, SCC, or KA and no indication to surgery or RT. MTX was injected subcutaneously proceeding from the periphery

\section{G. Argenziano - C. M. Giorgio}

Dermatology Unit, University of Campania, Naples, Italy

C. Requena · E. Nagore · O. Sanmartín

Department of Dermatology, Instituto Valenciano

de Oncología, València, Spain

E. Nagore · O. Sanmartín

School of Medicine, Universidad Católica de Valencia San Vicente Mártir, València, Spain

A. Martorell

Department of Dermatology, Hospital de Manises, València, Spain

F. Fantini

Department of Dermatology, Ospedale "Manzoni" Lecco, Lecco, Italy 
of the lesion toward the center. Different protocols in terms of dose, frequency, and length of treatment were used to compare them. Treatment efficacy was evaluated in terms of tumor size reduction. Patients were divided into three groups: responders (improvement of more than $50 \%)$, partial responders $(<50 \%)$, and non-responders (no improvement or worsening). All data were analyzed using the chi-squared test $\left(\chi^{2}\right)$.

Results: Thirty-five patients were included. Twenty-one patients suffered from SCC, 12 from KA, and 2 from BCC. KA showed a higher response rate than SCC and BCC. For AK, 92\% of patients had a complete resolution; $8 \%$ were partial responders. For SCC, $47.6 \%$ of cases were responders and $14.3 \%$ partial responders, while $38 \%$ non-responders. All BCCs showed no improvement. A treatment protocol of weekly injections, performed for 4 to 6 weeks, was the most efficient. Doses of $25 \mathrm{mg} / \mathrm{ml}$ per session seemed to be most effective. About one third of our patients developed side effects with mild anemia being the most frequent.

Conclusions: For selected cases, intralesional MTX can be a safe and effective option for the treatment of KT, especially in case of KA and, to a lesser extent, SCC.

Keywords: Basal cell carcinoma; Intralesional methotrexate; Kratinocytic tumors; Keratoacanthoma; Non-melanoma skin cancer; Squamous cell carcinoma; Surgery

\section{Key Summary Points}

\section{Why carry out this study?}

Keratinocyte tumors are the most frequent human tumors found in medical pracice. A surgical approach is not always the best option, and radiotherapy, as well as systemic chemotherapy, comes with a high burden of costs and side effects

Intralesional chemotherapy, in particular with MTX, could be a conservative yet effective treatment option

\section{What was learned from the study?}

Keratoacanthoma (KA) response rate to intralesional MTX is excellent. All KAs in our study improved partially or completely. About one half of squamous cell carcinomas showed either improvement or resolution, while basal cell carcinoma had no improvement

A treatment protocol consisting of weekly injections, performed for 4-6 weeks, seemed to be the most efficient. Dosages $\leq$ $20 \mathrm{mg} / \mathrm{ml}$ per session showed little efficacy compared with $25 \mathrm{mg} / \mathrm{ml}$ doses. The latter appeared more effective than $50 \mathrm{mg} / \mathrm{ml}$ as well

For selected cases, intralesional MTX can be a safe and effective option for the treatment of KT, especially in case of keratoacanthomas and, to a lesser extent, SCC

\section{INTRODUCTION}

Non-melanoma skin cancer (NMSC) is the most common malignancy worldwide [1]. The vast majority of NMSCs are keratinocyte tumors (KT). In particular, squamous cell carcinoma (SCC) and basal cell carcinoma (BCC) are the two most frequent sub-types, with BCC accounting for almost $80 \%$ of the cases of KT, while SCC for $20 \%$ [2-3]. Sun exposure is the main risk factor for the development of these tumors, especially in case of SCC [4]. In 2002, the incidence of SCC in Australia, where the highest number of cases is registered, was 387 cases per 100,000, while in Europe the highest rates were reported in Wales (31.7 cases per 100,000 person/year) and Switzerland (28.9 cases per 100,000 person/year) [5-6]. In Italy, AIRTUM (Associazione Italiana Registro Tumori) reported 127,879 new cases of skin cancer in a 10-year period (2005-2015) [7]. BCCs and SCCs are common, but fortunately the prognosis is good in the vast majority of cases. While risk of BCC metastasizing is extremely low, SCC is more aggressive, yet it is associated with a 5 -year median survival rate $>90 \%$. Metastases 
develop in $2-5 \%$ of SCC cases [6]. If metastatic lesions develop, the median survival is $<2$ years. NMSC is the fifth most expensive cancer for the US health care system. This economic burden depends on the high incidence of $\mathrm{KT}$, recurrence rates and cost of individual treatments [8].

Nowadays, the gold standard treatment for NMSC is surgery. However, in particular cases, surgery may not be the optimum choice. Several considerations have to be taken into account when deciding whether to perform a surgical procedure: first, patient features such as age and comorbidities, then tumor characteristics such as lesion size and anatomic location. Finally, the surgical procedure itself has to be considered as well: the risk of recurrence, infection, or excessive bleeding must guide the surgeon while deciding about the best treatment option for each patient.

When surgery is not indicated, few effective therapeutic options are available. Radiotherapy (RT) is widely used and seems to be quite effective as well. Unfortunately, RT is time consuming and expensive. Frequent treatments are often demanding for the patient, who has to come to the hospital many days in a few weeks. Moreover, RT can determine the secondary development of radiation dermatits. For larger tumors, RT is used as neoadiuvant therapy, but it is not indicated for the treatment of extensive areas, especially on prominent sites such as the face.

Systemic treatments directed against molecular targets have recently given promising results. Smoothened (Smo) receptor inhibitors, such as vismodegib and sonidegib, can be effective therapeutic options for locally advanced BCC. Likewise, anti-PD-1 drugs such as cemiplimab-and to a lesser extent antiEGFr-were proven to be effective in treating SCC. However, these treatments are only able to completely eradicate the tumor in a minority of patients. Other subjects show a partial response, which can be held only with a prolonged maintenance treatment. These long periods of CHT are associated with a high risk of toxicity, often unbearable for many patients. In addition, CHT has to be frequently discarded because of contraindications.

To avoid invasive surgery approaches or the frequent side effects related to systemic drugs, intralesional chemotherapy (iCHT) can be a conservative yet effective alternative.

Methotrexate (MTX) is widely used in oncology as an anti-tumoral agent. Its mechanisms of action include the competitive inhibition of the folic acid reductase enzyme, which is essential to supply methyl donor groups for DNA, RNA, and protein synthesis. This inhibition enables the conversion of dihydrofolic to tetrahydrofolic acid, blocking the production of thymidylic acid, a pyrimidine metabolite. Without thymidylic acid, DNA synthesis is impossible and cell division is therefore arrested [9].

The aim of the study was to retrospectively evaluate the effectiveness and safety of intralesional chemotherapy with MTX for the treatment of squamous cell carcinoma (SCC), keratoacanthoma (KA), and basal cell carcinoma (BCC).

MTX was only administered to patients who had contraindications to surgery, RT, surgery, or systemic drug therapy. The secondary objective of the study was to compare different treatment protocols in terms of dose and duration of treatment.

\section{METHODS}

This is a multicenter retrospective study: the Departments of Dermatology of Brescia, Chieti, L'Aquila, Lecco, and Napoli in Italy performed this study together with the Instituto Valenciano de Oncología of Valencia in Spain. All patients, included in our study between 2017 and 2019, had a histologically confirmed diagnosis of BCC, SCC, or KA (exclusively eruptive squamous cell carcinoma with keratoacanthoma-like features). Surgery was considered as the first option followed by RT. Considering the parameters previously mentioned (age, comorbidities, lesion size and anatomic location, risk of recurrence, infection, etc.), an alternative treatment was needed for each patient. Therefore, it was decided to treat them with intralesional MTX. Patients refusing surgery and RT, and subjects previously treated with such techniques that later showed a recurrence or disease progression, were included as well. Before including a patient in the study, each case was subjected to a multidisciplinary board 
consultation. Exclusion criteria included: bone marrow and hepatic failure; HIV, HBV, and $\mathrm{HCV}$ infection; and metastasis. Information about age, sex, and tumor size and site was collected, along with information about treatment protocols (cumulative dose, length, number of procedures). All patients were thoroughly informed and gave their consent to treatment. Moreover, the study was approved by the Internal Scientific Board of Dermatology of the Department of Medicine and Aging Science, University G. D'Annunzio, Chieti Pescara, Italy. This study was performed in accordance with the Helsinki Declaration of 1964 and its later amendments. The patients also provided their written informed consent for the publication of their images.

A physical examination was performed before each administration of MTX to assess the presence of side effects and the response to treatment, which was evaluated through the measurement of the main axis of the tumor. All patients underwent a laboratory workup with hematology, liver and kidney biochemistries, and urine analysis before and a month after the treatment. Folic acid supplementation was administered in accordance with guidelines to avoid the risk of development of hematologic side effects. MTX was injected subcutaneously, using a syringe with a 30-gauge needle. The tumor was divided into four equal parts. The drug was equally distributed in the four parts, treating the periphery of each area with a single injection (four injections per lesion per treatment session). At the following visit, if the tumor showed a decrement is size, injections were performed at the new periphery of the lesion, proceeding in this way from the outer parts toward the center. As there is no standardized protocol available at the moment, each physician used a different protocol in terms of dose, frequency, and length of treatment. The chosen dose and time interval between each administration were kept the same for each patient. This allowed us to compare different regimens. Treatment efficacy was evaluated in terms of reduction in size of the tumor at different time points: 3 and 6 months. Regarding the response to therapy, patients were divided into three groups: responders (improvement $>50 \%$ ), partial responders (improvement $<50 \%$ ), and non-responders (no improvement or worsening of the lesion). All data were analyzed using the chi-squared test $\left(\chi^{2}\right)$ to search for a statistically relevant correlation between the different factors considered.

\section{RESULTS}

Thirty-five patients were included in this study. The average age was $79.57 \pm 11.02$ years. Seventeen patients were male; 18 were female. Twenty-one patients suffered from SCC, 12 from KA, and 2 from BCC. The average dimension before treatment (major axis) was $3.6 \pm 2.61 \mathrm{~cm}$. Two patients had been previously treated with cryotherapy and one with RT. The average number of treatments was $4.46 \pm 2.15$, while its duration ranged from 3 to 16 weeks, averaging $6.54 \pm 2.84$ weeks. Cumulative average dose was $133.29 \pm 99.24 \mathrm{mg} / \mathrm{ml}$, while average dose for a single treatment was $22.15 \pm 15.6 \mathrm{mg} / \mathrm{ml}$. Twenty-one patients were responders, four had a partial response, and ten patients were non-responders (Table 1).

The initial dimension of the tumor does not seem to influence the efficacy of the treatment. Indeed, partially improved lesions had a larger initial size than lesions that showed no response. A treatment protocol consisting of weekly injections, performed for 4-6 weeks, seemed to be the most efficient. After the 6th week, the treatment appeared to lose its efficacy, and no further beneficial effect was observed. Dosages $\leq 20 \mathrm{mg} / \mathrm{ml}$ per session showed little efficacy compared with doses of $25 \mathrm{mg} / \mathrm{ml}$. The latter appeared more effective than $50 \mathrm{mg} / \mathrm{ml}$ as well (Table 2). No statistically significant differences were found when comparing the efficacy of the treatment for tumors in different areas $(p=0.580)$. Similarly, there was no difference in efficacy between the immune-suppressed patient and the others. Previous treatments also did not seem to influence the efficacy of this therapy. All patients who had already been treated with other techniques showed either a complete or partial response. 
Table 1 Different responses of KA, SCC and BCC to different treatment protocols

\begin{tabular}{lllllll}
\hline Variable & $\boldsymbol{N}$ & Overall & $\begin{array}{l}\text { NR }(\boldsymbol{n}=\mathbf{1 0}) \\
\text { Mean } \pm \text { SD }\end{array}$ & $\mathbf{R P}(\boldsymbol{n}=\mathbf{4})$ & $\boldsymbol{R}(\boldsymbol{n}=\mathbf{2 1})$ & $\boldsymbol{p}$ value \\
\hline $\begin{array}{llllll}\text { Age } \\
\text { Sex }\end{array}$ & 35 & $79.57 \pm 11.01$ & $80.8 \pm 11.05$ & $75.5 \pm 13.33$ & $79.76 \pm 10.96$ & 0.590 \\
$\quad$ Male & 35 & 17.00 & $4(0.24)$ & $3(0.18)$ & $10(0.59)$ & 0.802 \\
Female & & 18.00 & $6(0.33)$ & $1(0.06)$ & $11(0.61)$ & \\
Weeks & 35 & $6.54 \pm 2.84$ & $5.5 \pm 2.37$ & $10 \pm 5.83$ & $6.38 \pm 1.77$ & 0.168 \\
Diameter & 35 & $3.6 \pm 2.61$ & $3.86 \pm 3.49$ & $6.5 \pm 3$ & $2.92 \pm 1.6$ & 0.433 \\
No. of treatments & 35 & $4.46 \pm 2.15$ & $3.4 \pm 1.51$ & $6.25 \pm 1.5$ & $4.62 \pm 2.29$ & 0.670 \\
Cumulative dosage & 35 & $133.29 \pm 99.24$ & $96.5 \pm 67.78$ & $198.75 \pm 63.29$ & $138.33 \pm 111.86$ & 0.442 \\
Dosage for treatment & 35 & $22.15 \pm 15.6$ & $20.93 \pm 16.87$ & $30 \pm 23.18$ & $21.23 \pm 13.83$ & 0.141 \\
KT types & & & & & & \\
SCC & 35 & 21.00 & $8(0.38)$ & $3(0.14)$ & $10(0.48)$ & 0.018 \\
KA & & 12.00 & $0(0)$ & $1(0.08)$ & $11(0.92)$ & \\
BCC & 2.00 & $2(1)$ & $0(0)$ & $0(0)$ & \\
\hline
\end{tabular}

Table 2 Response to different single doses

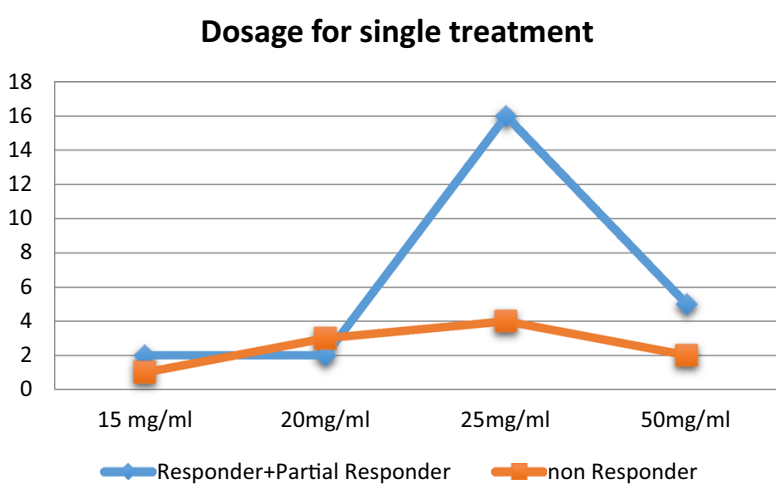

KA showed a higher response to intralesional MTX compared with SCC and BCC. All KAs improved after therapy: $92 \%$ had a complete resolution while $8 \%$ were partial responders. For SCC, $47.6 \%$ of patients were responders; $14.3 \%$ had a partial response, while $38 \%$ showed either no improvement or progression of the disease. All BCCs in our study showed no improvement (Figs. 1, 2, 3).
All patients showing a partial response, as the size of the tumor decreased, were then successfully treated with surgery. As each patient included in the study was at least initially unsuitable for surgery, a surgical approach was still not an option for non-responders. Therefore, non-responders were addressed to the oncologist to undergo systemic chemotherapy. Follow-up duration was 6 months. Neither patients showing a complete response nor the ones with partial response subsequently treated with surgery showed recurrence at 6 months. No data are available regarding non-responders as they were referred to another unit.

About one third of our patients developed side effects $(34.3 \%)$. Anemia $(\mathrm{Hb}<12 \mathrm{~g} / \mathrm{dl})$ was the most frequent (5 patients), but only one patient developed severe anemia and required a transfusion. Other common side effects observed were: leukopenia (1 patient), elevation of liver transaminases (4 patients), and increased nitrogen levels ( 1 patient). Non-responders developed side effects in $30 \%$ of cases, responders in 33\%, showing no difference between the two categories. Efficacy of 

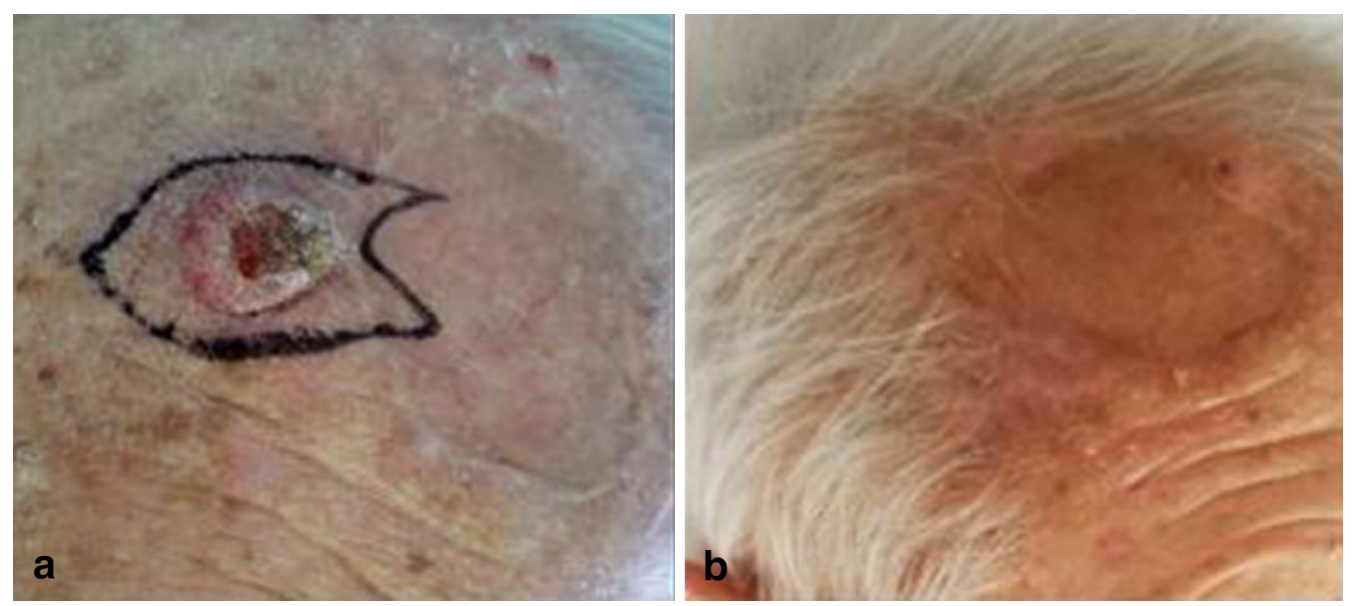

Fig. 1 Keratoacanthoma before treatment (a) showing a complete response after therapy (b)
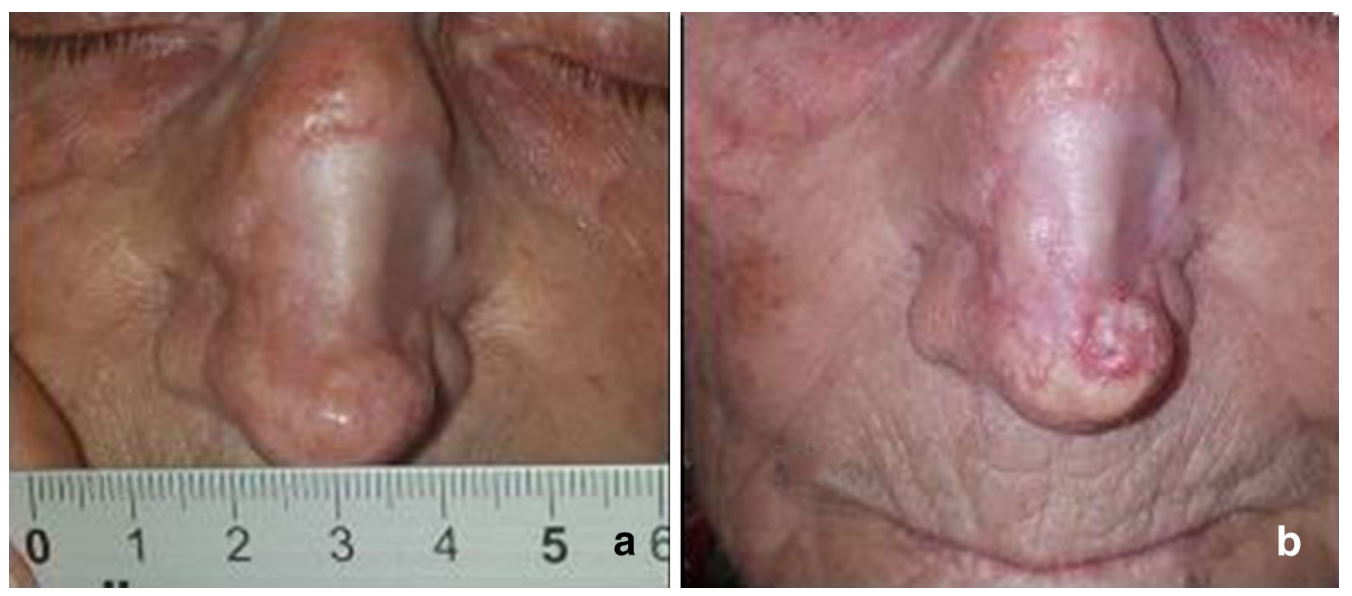

Fig. 2 Basal cell carcinoma before therapy (a) showing progression despite therapy (b)
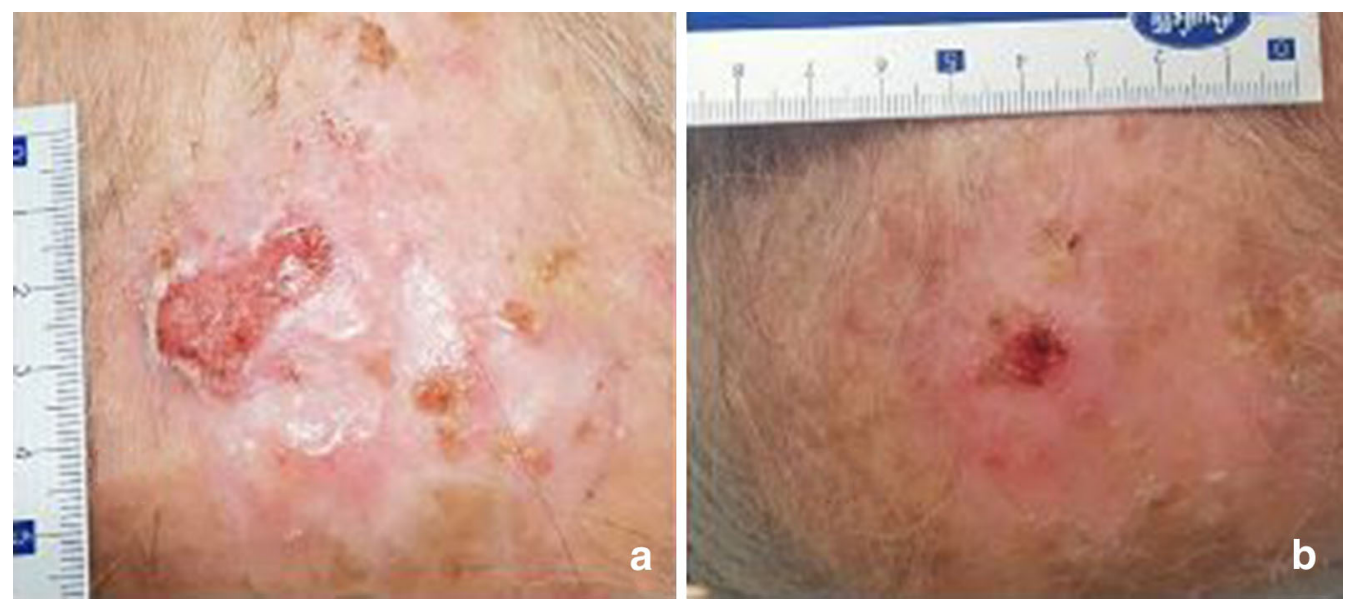

Fig. 3 Squamous cell carcinoma before therapy (a) showing a partial response to therapy (b) 
treatment does not seem to have a correlation with the development of side effects. Similarly, cumulative dose and duration of treatment did not seem to play a role. Two patients developed metastasis during treatment.

\section{DISCUSSION}

KTs are the most frequent human tumors observed in medical practice. Surgery is the gold standard for treating such lesions. As discussed before, in some cases a surgical approach might not be the best option. Patient and tumor features, along with cost and duration of the therapy, should be considered.

Even if the percentage of patients with KT who have some contraindication to surgery is low, the high frequency of KT determines a large number of patients for whom an alternative to surgery is needed. RT, which shows a high efficacy rate in the literature (up to $92 \%$ ), is an expensive option and is often quite demanding and time consuming for the patient. To the best of our knowledge, no study comparing surgery with RT is currently present in the literature. RT is mostly used as neoadjuvant therapy before surgery (to decrease the size of the tumor before surgical intervention) or in case of relapse. The risk of inducing a radiodermatitis, and therefore potentially another neoplastic lesion, and the complexity of treating a relapse if developed on an area previously treated with RT are the main critical issues when approaching this technique. Systemic CHT can be effective in treating advanced KT. However, complete eradication of the tumor is rare, and patients have to go through prolonged maintenance treatment, which carries a high risk of toxicity.

Intralesional MTX for the treatment of KT is described in the literature for $\mathrm{KA}$, often on a low number of patients. In our study, the efficacy of intralesional MTX was evaluated on 35 patients affected by different kinds of KT and not just KA. Several treatment protocols were compared as well. Our sample consisted of elderly, and therefore fragile, patients (average age 79.5 years). Most of the lesions developed on high-risk areas, such as the head or hands and feet. Our data show great efficacy for KAs, as all patients improved or were completely healed. About half of the patients with cSCC responded to therapy, while no patient affected by BCC showed any improvement. This difference might be explained by the mechanism through which MTX dispatches its pharmacologic properties. As MTX inhibits DNA synthesis during cell replication, rapidly growing tumors, such as KA and cSCC, are more sensitive to such therapy compared with slow-growing tumors such as BCC. This general consideration must take into account the reduced significance of the BCC data, given the low number of cases included. Our data regarding the correlation between the efficacy of intralesional MTX and the size of the tumor seem to confirm this hypothesis as well. As larger tumors likely replicate faster, they show a higher sensitivity to MTX than smaller lesions, which do not replicate at such a pace. A review from 2019 demonstrated a high efficacy of intralesional MTX for the treatment of KA (94\%) [10]. Our study confirms these data. In another study, Moss and Weber treated 157 KAs with a complete resolution in $88 \%$ of the cases [11]. There are few studies in the literature on the efficacy of MTX for treating cSCC. Moreover, most of them evaluate MTX efficacy as adjuvant therapy, but not as a solitary treatment. In 2016, Salido-Vallejo et al. compared 43 patients treated with surgery alone with 43 patients treated with intralesional MTX before a surgical approach. All surgical procedures were performed within 30 days of the diagnosis. In the first group, the average increment in lesion size was $19 \%$. Patients treated with MTX showed a decrease in lesion size of $23 \%$. Moreover, SCC of the lips seemed more responsive than tumors of other areas [12]. Similarly, in 2018 Sendin and Perez described a group of patients affected by SCC of the lips, treated with two injections of MTX 50 days before surgery. The size of these tumors decreased significantly $(68.18 \%$ decrease for the minor axis and $57.28 \%$ for the major) [13]. All the other studies on this matter are case reports in which MTX was again used as neoadjuvant therapy. Our study shows that MTX can be effective for about half of the cases of cSCC. This confirms that MTX can be a useful 
tool to treat tumors for which surgery could not be indicated. Regarding BCC, both lesions treated in our study showed an initial and mild improvement. There is only one study in the literature in which MTX was tested for the treatment of BCC. In this study, 11 patients were treated with a single dose of intralesional MTX; however, no improvement was observed [14]. One of the main issues regarding the use of intralesional MTX is that no specific standardized treatment protocol has been established yet. Our data suggest that weekly administration of at least $25 \mathrm{mg} / \mathrm{ml}$ for a 4-6-week period, might be the best approach. No improvement was observed after the 6 th week of treatment for any patient. No correlations between dose or duration of the treatment and side effects were found. Likewise, previous treatments, immune suppressive concomitant therapies, or site of the lesion do not seem to influence the efficacy of this therapy.

The main limitation of the study is the low number of patients affected by BCC.

\section{CONCLUSION}

For selected cases, intralesional MTX can be a safe and effective option for the treatment of $\mathrm{KT}$, especially in case of KA and, to a lesser extent, SCC. Moreover, this procedure can also be considered as a pre-surgical option to reduce the tumor size and make the surgical procedure easier and safer for the patient.

\section{ACKNOWLEDGEMENTS}

Funding. No funding or sponsorship was received for this study or publication of this article.

Authorship. All named authors meet the International Committee of Medical Journal Editors (ICMJE) criteria for authorship for this article, take responsibility for the integrity of the work as a whole, and have given their approval for this version to be published.
Disclosures. Giulio Gualdi, Simone Caravello, Fabiano Frasci, Federica Giuliani, Ruggero Moro, Maria C. Fargnoli, Valeria Ciciarelli, Giuseppe Argenziano, Caterina M. Giorgio, Celia Requena, Eduardo Nagore, Onofre Sanmartín, Fabrizio Fantini, and Paolo Amerio have nothing to disclose. Piergiacomo CalzavaraPinton is a memeber of the journal's Editorial Board.

Compliance with Ethics Guidelines. All patients were thoroughly informed and gave their consent to treatment. Moreover, the study was approved by the Internal Scientific Board of Dermatology of the Department of Medicine and Aging Science, University G. D'Annunzio, Chieti Pescara, Italy. This study was performed in accordance with the Helsinki Declaration of 1964 and its later amendments. The patient also provided their written informed consent for the publication of their images.

Data Availability. The datasets during and/ or analyzed during the current study are available from the corresponding author on reasonable request.

Open Access. This article is licensed under a Creative Commons Attribution-NonCommercial 4.0 International License, which permits any non-commercial use, sharing, adaptation, distribution and reproduction in any medium or format, as long as you give appropriate credit to the original author(s) and the source, provide a link to the Creative Commons licence, and indicate if changes were made. The images or other third party material in this article are included in the article's Creative Commons licence, unless indicated otherwise in a credit line to the material. If material is not included in the article's Creative Commons licence and your intended use is not permitted by statutory regulation or exceeds the permitted use, you will need to obtain permission directly from the copyright holder. To view a copy of this licence, visit http:// creativecommons.org/licenses/by-nc/4.0/. 


\section{REFERENCES}

1. Parekh V, Seykora JT. Cutaneous squamous cell carcinoma. Clin Lab Med. 2017;37(3):503-25. https://doi.org/10.1016/j.cll.2017.06.003 (PubMed PMID: 28802498).

2. Que SKT, Zwald FO, Schmults CD. Cutaneous squamous cell carcinoma: incidence, risk factors, diagnosis, and staging. J Am Acad Dermatol. 2018;78(2):237-47. https://doi.org/10.1016/j.jaad. 2017.08.059 (PubMed PMID: 29332704).

3. Nehal KS, Bichakjian CK. Update on keratinocyte carcinomas. N Engl J Med. 2018;379(4):363-74. https://doi.org/10.1056/NEJMra1708701 (PubMed PMID: 30044931).

4. Apalla Z, Calzavara-Pinton P, Lallas A, et al. Histopathological study of perilesional skin in patients diagnosed with nonmelanoma skin cancer. Clin Exp Dermatol. 2016;41(1):21-5.

5. Staples MP, Elwood M, Burton RC, et al. Non-melanoma skin cancer in Australia: the 2002 national survey and trends since 1985. Med J Aust. 2006;184(1):6-10 (PubMed PMID: 16398622).

6. Peris K, Alaibac M, Argenziano G, et al. Cutaneous squamous cell carcinoma. Italian Guidelines by SIDeMaST adapted to and updating EADO/EDF/ EORTC guidelines. G Ital Dermatol Venereol. 2018;153(6):747-62. https://doi.org/10.23736/ S0392-0488.18.06093-5 (Epub 2018 Jun 11. PubMed PMID: 29898593).

7. Giovanna T, Enrico C, Vincenzo C et al (2018) Analisi epidemiologica e caratterizzazione morfologica di pazienti affetti da tumore della cute non melanoma da registro nazionale AIRTUM.
8. Gualdi G, Monari P, Apalla Z, et al. Surgical treatment of basal cell carcinoma and squamous cell carcinoma. G Ital Dermatol Venereol. 2015;150(4): 435-47 (Epub 2015 Jul 3 PubMed PMID: 26140396).

9. Lester RS. Methotrexate. Clin Dermatol. 1989;7(3): 128-35 (PubMed PMID: 2680016).

10. Kiss N, Avci P, Bánvölgyi A, et al. Intralesional therapy for the treatment of keratoacanthoma. Dermatol Ther. 2019;32(3):12872. https://doi.org/ 10.1111/dth.12872 (Epub 2019 Apr 4. Review. PubMed PMID: 30866135).

11. Moss M, Weber E, Hoverson K, et al. Management of keratoacanthoma: 157 tumors treated with surgery or intralesional methotrexate. Dermatol Surg. 2019;45(7):877-83. https://doi.org/10.1097/DSS. 0000000000001739 (PubMed PMID: 30608293).

12. Salido-Vallejo R, Garnacho-Saucedo G, SánchezArca $M$, et al. Neoadjuvant intralesional methotrexate before surgical treatment of invasive squamous cell carcinoma of the lower lip. Dermatol Surg. 2012;38(11):1849-50. https://doi.org/10. 1111/j.1524-4725.2012.02540.x (Epub 2012 Aug 3 PubMed PMID: 22861167).

13. Bergón-Sendín M, Pulido-Pérez A, Suárez-Fernández R. Neoadjuvant intralesional methotrexate in squamous cell carcinoma of the lip. Australas J Dermatol. 2019;60(2):158-60. https://doi.org/10. 1111/ajd.12946 (Epub 2018 Oct 24. PubMed PMID: 30357801).

14. Balighi K, Ansari MS, Mirzaiepour M, et al. Treatment of basal cell carcinoma: is intralesional methotrexate an option? J Dermatolog Treat. 2018;29(8):745-6. https://doi.org/10.1080/ 09546634.2018.1451817 (Epub 2018 Mar 28. PubMed PMID: 29528258). 